Evidence for protection of the $\alpha$-chains by their chaperone. J. Biol. Chem. 282:6398-6404.

10. Kong, Y., et al. 2004. Loss of $\alpha$-hemoglobin-stabilizing protein impairs erythropoiesis and exacerbates $\beta$-thalassemia. J. Clin. Invest. 114:1457-1466. doi:10.1172/JCI200421982

11. Yu, X., et al. 2007. An erythroid chaperone that facilitates folding of $\alpha$-globin subunits for hemoglobin synthesis. J. Clin. Invest. 117:1856-1865. doi:10.1172/JCI31664.

12. dos Santos, C.O., Duarte, A.S., Saad, S.T., and Costa, F.F. 2004. Expression of $\alpha$-hemoglobin stabilizing protein gene during human erythropoiesis. Exp. Hematol. 32:157-162.
13. Senok, A.C., et al. 1997. Invasion and growth of Plasmodium falciparum is inhibited in fractionated thalassaemic erythrocytes. Trans. R. Soc. Trop. Med. Hyg. 91:138-143.

14. Viprakasit, V., et al. 2004. Evaluation of $\alpha$ hemoglobin stabilizing protein (AHSP) as a genetic modifier in patients with $\beta$ thalassemia. Blood. 103:3296-3299.

15. Lai, M.I., et al. 2006. $\alpha$-Haemoglobin stabilising protein is a quantitative trait gene that modifies the phenotype of $\beta$-thalassaemia. Br. J. Haematol. 133:675-682.

16. Vasseur-Godbillon, C., Marden, M.C., Giordano, P., Wajcman, H., and Baudin-Creuza, V. 2006
Impaired binding of AHSP to $\alpha$ chain variants: $\mathrm{Hb}$ Groene Hart illustrates a mechanism leading to unstable hemoglobins with $\alpha$ thalassemic like syndrome. Blood Cells Mol. Dis. 37:173-179.

17. Gallagher, P.G., Liem, R.I., Wong, E., Weiss, M.J., and Bodine, D.M. 2005. GATA-1 and Oct-1 are required for expression of the human $\alpha$-hemoglobin-stabilizing protein gene. J. Biol. Chem. 280:39016-39023.

18. Pilon, A.M., et al. 2006. Alterations in expression and chromatin configuration of the $\alpha$ hemoglobin-stabilizing protein gene in erythroid Kruppel-like factor-deficient mice. Mol. Cell. Biol. 26:4368-4377

\title{
Torn apart: membrane rupture in muscular dystrophies and associated cardiomyopathies
}

\author{
Jan Lammerding and Richard T. Lee
}

Cardiovascular Division, Department of Medicine, Brigham and Women's Hospital, Harvard Medical School, Boston, Massachusetts, USA.

\begin{abstract}
Muscular dystrophies are often caused by mutations in cytoskeletal proteins that render cells more susceptible to strain-induced injury in mechanically active tissues such as skeletal or cardiac muscle. In this issue of the JCI, Han et al. report that dysferlin participates in membrane resealing in cardiomyocytes and that exercise results in increased membrane damage and disturbed cardiac function in dysferlin-deficient mice (see the related article beginning on page 1805). Thus, in addition to repetitive membrane damage, inadequate membrane repair may participate in the pathogenesis of muscular dystrophies and cardiomyopathies.
\end{abstract}

Given the large deformations of many tissues such as skeletal muscle, it's remarkable that cells don't just tear apart. In fact, membrane rupture occurs frequently under physiological conditions in mechanically active tissues and can be experimentally demonstrated both in vivo and in vitro (1-3). Membrane rupture leads to loss of cytoplasmic components and allows the influx of extracellular ions, thus disturbing the carefully maintained ion balance between the cytoplasm and the extracellular space. To prevent cell death from local membrane damage, cells have mechanisms to preserve membrane integrity and to reseal or repair ruptured membranes. In the presence of high extracellular $\mathrm{Ca}^{2+}$ concentrations, injured nucleated cells reseal within 10-30 seconds (3).

\section{Membrane-repair mechanism(s)}

Membrane rupture leads to exposure of hydrophobic phospholipids to the aque-

Conflict of interest: The authors have declared that no conflict of interest exists.

Citation for this article: J. Clin. Invest. 117:1749-1752 (2007). doi:10.1172/JCI32686. ous environment, an energetically unfavorable state. However, the entropic forces that draw the membrane ends together are insufficient to reseal membrane lesions larger than $1 \mu \mathrm{m}$ in nucleated cells under physiological conditions (3), as membrane tension (driven by interaction of phospholipids with the underlying cytoskeleton) slows or completely blocks self sealing. Instead, cells utilize an active membrane-repair process based on active trafficking of endomembrane vesicles to the damage site and subsequent fusion with the plasma membrane by exocytosis, but many of the molecular details of this process remain unclear. It appears that membrane repair involves both a reduction in membrane tension - possibly by local depolymerization of the cortical cytoskeleton - and patch formation. In the latter process, homotypic fusion of membrane vesicles creates a patch at the rupture site that then fuses with the plasma membrane in a $\mathrm{Ca}^{2+}$-dependent process (Figure 1). Depending on the cell type, the vesicular membrane compartments participating in the repair may include cortical granules, yolk granules, endocytic components, lysosomes, and enlargosomes; the specific contribution of each is not always clear $(3,4)$.

The active membrane fusion process requires several membrane proteins, including SNARE proteins (a family of transmembrane proteins essential in most intracellular membrane fusion processes) and synaptotagmins (transmembrane proteins containing two highly conserved $\mathrm{Ca}^{2+}$-binding domains that are thought to serve as $\mathrm{Ca}^{2+}$ sensors; ref. 5). Most recently, ferlins have been identified as a conserved protein family that participate in membrane repair. The ferlin family consists of four different genes that encode dysferlin, myoferlin, otoferlin, and Fer1L4; dysferlin-null cells show accumulation of membrane vesicles near the damaged membrane $(3,4)$.

\section{Membrane disruption leads to muscular dystrophy}

Membrane rupture is most prevalent in skeletal and cardiac muscle; up to $25 \%$ of muscle cells show signs of transient membrane damage under physiological conditions (1-3). Higher organisms have specialized tissue and cell architectures that minimize the stress placed on the plasma membrane by directly transmitting forces from the extracellular matrix to the cytoskeleton through dedicated protein complexes, such as the dystrophin-glycoprotein complex, and thus shielding the fragile plasma membrane. Not surprisingly, mutations that disrupt these stress-bearing elements render cells 


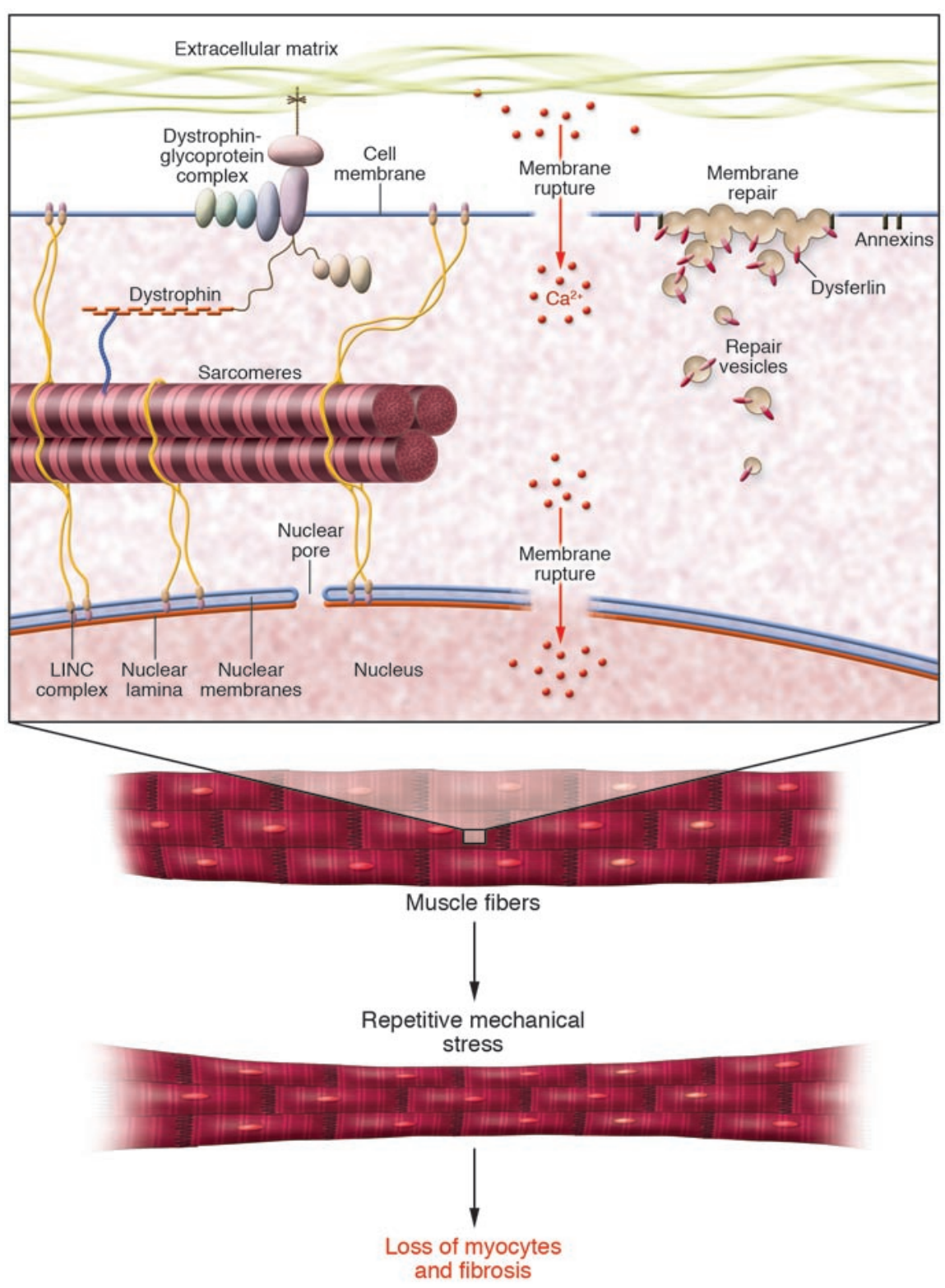

more susceptible to contraction-induced injury and can result in muscular dystrophy or cardiomyopathy. Recent work by Kevin Campbell's group has demonstrated that defective membrane repair can provide an alternative mechanism for muscular dystrophies (6). Dysferlin-null mice suffer from impaired membrane repair and develop progressive muscular dystrophy and cardiomyopathy, even though these animals have stable and functional dystrophin-glycoprotein complexes and show no increase in membrane damage during eccentric contraction. In humans, dysferlin mutations cause limbgirdle muscular dystrophy type $2 \mathrm{~B}$ and Miyoshi myopathy $(7,8)$.
Most studies of muscular dystrophies have focused on the cellular damage in skeletal muscle, but it is important to recognize that several types of muscular dystrophies affect cardiac muscle and that cardiac failure is responsible for a large number of deaths in Duchenne muscular dystrophy and Emery-Dreifuss muscular dystrophy patients $(9,10)$. In this issue of the JCI, Han et al. (11) report that dysferlin-mediated membrane repair is critical to protection of cardiomyocytes from stressinduced injury. Han and colleagues applied a sophisticated in vitro assay to directly demonstrate that $\mathrm{Ca}^{2+}$-dependent membrane repair is impaired in dysferlin-null cardiomyocytes. Next, they demonstrated

\section{Figure 1}

Repetitive mechanical strain causes rupture in the plasma membrane under physiological conditions. Mutations that predispose cells to membrane damage or impair the normal repair process cause accumulation of necrotic cells in mechanically active tissue, resulting in muscular dystrophies and cardiomyopathies. Mutations in the nuclear envelope proteins lamin $\mathrm{A} / \mathrm{C}$ or emerin can decrease the stability of the nuclear lamina and result in nuclear envelope rupture, causing similar muscular dystrophies and cardiomyopathies. In this issue of the $\mathrm{JCl}$, Han et al. (11) report that dysferlin is required for stress-induced membrane repair in cardiomyocytes. While the precise membrane-repair mechanism remains unclear, it is thought that dysferlin-carrying repair vesicles are recruited to the rupture site and fuse with the plasma membrane through interaction with annexins, other dysferlins, and other unknown binding partners (27). LINC, linker of nucleoskeleton and cytoskeleton. Figure adapted with permission from the New England Journal of Medicine (28). defective membrane repair in dysferlin-null mice in vivo in the heart following stress exercise and disturbed cardiac function. The notion that deficient membrane repair and increased membrane fragility are two independent mechanisms was supported in that damage was even more severe in animals bred by intercrossing dysferlinand dystrophin-null mice. These findings suggest an overarching concept underlying the majority of mutations implicated in muscular dystrophies and associated cardiomyopathy: Repetitive strain and contraction in mechanically active tissue cause progressive myocyte necrosis through cumulative damage in cells that cannot be sufficiently repaired. 


\section{Nuclear membrane damage can also cause muscular dystrophies}

The concept of repetitive membrane injury may not be limited to the cytoplasmic membrane but may also apply to a group of muscular dystrophies that arise from mutations in nuclear envelope proteins. Lamins are the main components of the nuclear lamina, a dense protein network underlying the inner nuclear membrane. Mutations in lamin A/C cause a plethora of human diseases, including Emery-Dreifuss muscular dystrophy, limb-girdle muscular dystrophy type 1B, and dilated cardiomyopathy (12). The molecular mechanisms responsible for the laminopathies remain unclear, but increased nuclear fragility could contribute to the muscle-specific phenotypes. Muscle biopsies from Emery-Dreifuss muscular dystrophy patients and from lamin $\mathrm{A} / \mathrm{C}$-null mice show characteristic nuclear damage, including protrusions of chromatin from the nucleus, loss of nuclear membrane, and even aggregation of mitochondria within the nucleoplasm (13-17). Cells deficient in lamin A/C have increased nuclear fragility under repetitive mechanical stress (18-21). The muscular laminopathies share several other features with the muscular dystrophies caused by defective plasma membrane structure or repair. Lamins interact with integral inner nuclear membrane proteins and with chromatin and are part of the linker of nucleoskeleton and cytoskeleton (LINC) complex connecting the nucleus to the cytoskeleton (Figure 1). Similar to dysferlin, lamin $\mathrm{A} / \mathrm{C}$ and emerin are ubiquitously expressed, but many of the related disease phenotypes are focused on skeletal and cardiac muscle.

Interestingly, the disease mechanisms of the nuclear envelope muscular dystrophies might not just mirror the pathophysiology in muscular dystrophies caused by plasma membrane damage. The cytoskeletal network and nuclear and plasma membrane proteins form a continuous physical connection that begins at the extracellular matrix and extends all the way to the nuclear interior, and disruption of any of these components could affect the other structures. Furthermore, in mice carrying a dominant-negative $\delta$-sarcoglycan mutation that results in dilated cardiomyopathy, $\delta$-sarcoglycan is mislocalized in cardiomyocytes, moving from the plasma membrane to the nucleus. This mislocalization is accompanied by partial nuclear sequestration of $\beta$ - and $\gamma$-sarcoglycans and mislocalization of lamin A/C and emerin from the nuclear envelope into the nucleoplasm (22).

\section{Treatment perspectives for muscular dystrophies}

There are currently no effective treatment options for patients suffering from muscular dystrophies. Recently, plasma membrane sealants such as poloxamer 188 have been shown to efficiently reduce cellular damage in muscular dystrophy and to improve cardiac function in dystrophin-deficient mice (23). However, poloxamer 188 failed to prevent exerciseinduced membrane damage in skeletal muscle fibers of $m d x$ mice, a mouse model of Duchenne muscular dystrophy lacking dystrophin (24). Furthermore, the most effective concentrations of membrane sealant are not well tolerated by healthy human volunteers even for short durations (25) while muscular dystrophy patients would require lifelong treatment, as membrane sealants are only effective when present during membrane rupture. The discovery of alternative, better-tolerated sealants or drugs that can drive the membranerepair process would represent significant advances. These therapies could benefit patients with a wide variety of muscular dystrophies, regardless of whether they are caused by increased membrane fragility, deficient repair, or both.

\section{Perspectives for future research}

The molecular mechanisms of the membrane-repair process are only now emerging, and it remains unclear what other molecules are necessary and critical for efficient membrane repair. Myoferlin in particular could be important for muscular dystrophies, as it is highly expressed in skeletal and cardiac muscle and is upregulated in skeletal muscle in dystrophin-deficient mice (26). Interestingly, myoferlin - in contrast to dysferlin - is also present in the nucleus of skeletal and cardiac muscle fibers, so it could also participate in nuclear envelope repair. In the plasma membrane, additional membrane must be added to the surface at or near the disruption site through fusion with cytoplasmic vesicles. In the nucleus, the endoplasmic reticulum is continuous with the outer nuclear membrane and provides a large membrane reservoir, but interaction between the inner nuclear membrane and the lamina could also require additional membrane-repair mechanisms and patch formation similar to the processes seen at the plasma membrane.

The study by Han et al. (6) highlights the concept that membrane injury, a normal process in mechanically active tissues such as muscle, must be met with adequate membrane repair. In the highly mechanically active milieu of skeletal and cardiac muscle, cumulative membrane damage may provide slow but progressive loss of cells with fibrotic replacement. Understanding the balance of membrane injury and repair may provide insight into the highly variable stages of penetrance in different muscle diseases as well as viable therapeutic strategies.

\section{Acknowledgments}

This work was supported by grants from the NIH (HL073809, HL081404, HL082792), the American Heart Association $(0635359 \mathrm{~N})$, and the Progeria Research Foundation.

Address correspondence to: Richard T. Lee, Brigham and Women's Hospital, 65 Landsdowne Street, Partners Research Facility, Cambridge, Massachusetts 02139, USA. Phone: (617) 768-8282; Fax: (617) 768-8270; E-mail: rlee@partners.org.

1. Clarke, M.S., Khakee, R., and McNeil, P.L. 1993. Loss of cytoplasmic basic fibroblast growth factor from physiologically wounded myofibers of normal and dystrophic muscle. J. Cell Sci. 106:121-133.

2. McNeil, P.L., and Khakee, R. 1992. Disruptions of muscle fiber plasma membranes. Role in exerciseinduced damage. Am. J. Pathol. 140:1097-1109.

3. McNeil, P.L., and Steinhardt, R.A. 2003. Plasma membrane disruption: repair, prevention, adaptation. Annu. Rev. Cell Dev. Biol. 19:697-731.

4. McNeil, P.L., and Kirchhausen, T. 2005. An emergency response team for membrane repair. Nat. Rev. Mol. Cell Biol. 6:499-505.

5. Giraudo, C.G., Eng, W.S., Melia, T.J., and Rothman, J.E. 2006. A clamping mechanism involved in SNARE-dependent exocytosis. Science. 313:676-680.

6. Bansal, D., et al. 2003. Defective membrane repair in dysferlin-deficient muscular dystrophy. Nature. 423:168-172.

7. Bashir, R., et al. 1998. A gene related to Caenorhabditis elegans spermatogenesis factor fer- 1 is mutated in limb-girdle muscular dystrophy type 2B. Nat. Genet. 20:37-42.

8. Liu, J., et al. 1998. Dysferlin, a novel skeletal muscle gene, is mutated in Miyoshi myopathy and limb girdle muscular dystrophy. Nat. Genet. 20:31-36.

9. Wessely, R., Seidl, S., and Schomig, A. 2005. Cardiac involvement in Emery-Dreifuss muscular dystrophy. Clin. Genet. 67:220-223.

10. Finsterer, J., and Stollberger, C. 2003. The heart in human dystrophinopathies. Cardiology. 99:1-19.

11. Han, R., et al. 2007. Dysferlin-mediated membrane repair protects the heart from stress-induced left ventricular injury. J. Clin. Invest. 117:1805-1813. doi:10.1172/JCI30848. 
12. Broers, J.L., Hutchison, C.J., and Ramaekers, F.C. 2004. Laminopathies. J. Pathol. 204:478-488.

13. Fidzianska, A., and Hausmanowa-Petrusewicz, I. 2003. Architectural abnormalities in muscle nuclei. Ultrastructural differences between X-linked and autosomal dominant forms of EDMD. J. Neurol. Sci. 210:47-51.

14. Fidzianska, A., Toniolo, D., and HausmanowaPetrusewicz, I. 1998. Ultrastructural abnormality of sarcolemmal nuclei in Emery-Dreifuss muscular dystrophy (EDMD). J. Neurol. Sci. 159:88-93.

15. Ostlund, C., and Worman, H.J. 2003. Nuclear envelope proteins and neuromuscular diseases. Muscle Nerve. 27:393-406

16. Sullivan, T., et al. 1999. Loss of A-type lamin expression compromises nuclear envelope integrity leading to muscular dystrophy. J. Cell Biol. 147:913-920.

17. Sylvius, N., et al. 2005. In vivo and in vitro examination of the functional significances of novel lamin gene mutations in heart failure patients. J. Med. Genet. 42:639-647.
18. Broers, J.L., et al. 2004. Decreased mechanical stiffness in LMNA-/- cells is caused by defective nucleo-cytoskeletal integrity: implications for the development of laminopathies. Hum. Mol. Genet. 13:2567-2580

19. Lammerding, J., et al. 2006. Lamins A and C but not lamin B1 regulate nuclear mechanics. J. Biol. Chem. 281:25768-25780.

20. Lammerding, J., et al. 2005. Abnormal nuclear shape and impaired mechanotransduction in emerin-deficient cells. J. Cell Biol. 170:781-791.

21. Lammerding, J., et al. 2004. Lamin A/C deficiency causes defective nuclear mechanics and mechanotransduction. J. Clin. Invest. 113:370-378. doi:10.1172/JCI200419670.

22. Heydemann, A., Demonbreun, A., Hadhazy, M., Earley, J.U., and McNally, E.M. 2007. Nuclear sequestration of delta-sarcoglycan disrupts the nuclear localization of lamin $\mathrm{A} / \mathrm{C}$ and emerin in cardiomyocytes. Hum. Mol. Genet. 16:355-363.

23. Yasuda, S., et al. 2005. Dystrophic heart failure blocked by membrane sealant poloxamer. Nature. 436:1025-1029.

24. Quinlan, J.G., et al. 2006. Poloxamer 188 failed to prevent exercise-induced membrane breakdown in mdx skeletal muscle fibers. Neuromuscul. Disord. 16:855-864.

25. Jewell, R.C., Khor, S.P., Kisor, D.F., LaCroix, K.A. and Wargin, W.A. 1997. Pharmacokinetics of RheothRx injection in healthy male volunteers. J. Pharm. Sci. 86:808-812.

26. Davis, D.B., Delmonte, A.J., Ly, C.T., and McNally, E.M. 2000. Myoferlin, a candidate gene and potential modifier of muscular dystrophy. Hum. Mol. Genet. 9:217-226.

27. Bansal, D., and Campbell, K.P. 2004. Dysferlin and the plasma membrane repair in muscular dystrophy. Trends Cell Biol. 14:206-213.

28. Dalakas, M.C., et al. 2000. Desmin myopathy, a skeletal myopathy with cardiomyopathy caused by mutations in the desmin gene. $N$. Engl. J. Med. 342:770-780.

\title{
p53, chemokines, and squamous cell carcinoma
}

\author{
David M. Owens
}

Departments of Dermatology and Pathology, Columbia University College of Physicians and Surgeons, New York, New York, USA.

\begin{abstract}
The genetic and epigenetic events underlying cutaneous squamous cell carcinoma (SCC) have been actively studied; however, no resulting preventative or therapeutic strategies have successfully targeted this lesion, apart from surgery. In this issue of the JCI, two novel regulators of SCC pathogenesis are introduced, gain-of-function mutations in the $p 53$ gene, reported by Caulin et al., and chemokine sequestration by the D6 receptor, reported by Nibbs et al. (see the related articles beginning on pages 1884 and 1893, respectively). These studies provide new twists and insights into the development of this potentially lethal disease.
\end{abstract}

Nonmelanoma skin cancer (NMSC) is the most common type of human malignancy, with over one million new cases in the United States annually (1-3). NMSC includes basal cell carcinoma and squamous cell carcinoma (SCC), with SCCs constituting approximately $20 \%$ of all NMSCs. In contrast to basal cell carcinomas, SCCs characteristically exhibit a high propensity for invasion and metastasis and may be lethal $(1,2)$. While the genetic and epigenetic events associated with SCC pathogenesis have been extensively studied, no resulting therapeutic approaches have been generated for the prevention or treatment of this potentially lethal disease. This, coupled with the escalating incidence of NMSC over the

Nonstandard abbreviations used: NMSC, nonmelanoma skin cancer; SCC, squamous cell carcinoma.

Conflict of interest: The author has declared that no conflict of interest exists.

Citation for this article: J. Clin. Invest. 117:1752-1755 (2007). doi:10.1172/JCI32719. last few decades, has made this disease a major public health issue.

\section{The $p 53$ oncogene: implications for cutaneous SCC}

It is widely accepted that genetic insults are indispensable in the formation of a frank tumor. In the development of human cutaneous SCCs, alterations in ras genes $(10 \%-30 \%$ incidence) $(3,4)$ and the $p 53$ tumor suppressor gene $(40 \%-50 \%$ incidence) $(5,6)$ have been most heavily implicated. While the majority of these lesions are missense mutations, the functional assessment of p53 missense mutations is complicated in that some give rise to a loss-of-function or null phenotype classically associated with tumor suppressor genes, whereas the majority of $p 53$ missense mutations appear to result in a gain-of-function phenotype. While the presence of both types of p53 mutations in SCCs denotes a selection advantage to these genetic lesions, whether gain-offunction or loss-of-function mutations are more critical for SCC development is unclear. Therefore, the understanding of p53 phenotype status, i.e., tumor suppressive versus oncogenic, as it relates to SCC formation and progression is of paramount importance for the development of relevant therapeutic approaches that target this gene.

In this issue of the JCI, Caulin and coworkers (7) address this problem with an elegant transgenic mouse skin model that delivers allelic doses of oncogenic $K$-ras (8) in combination with either $p 53$ gain-of-function or null mutations under the regulatory control of an inducible Cre recombinase and targeted to the proliferative layer of the epidermis by the cytokeratin K5 promoter. Using this approach, the authors clearly show that certain p53 missense mutations occurring in human SCCs demonstrate gainof-function properties in vivo and that p53 gain-of-function mutations accelerate both the frequency and progression of SCCs genetically initiated with oncogenic ras. For over a decade, the conventional regulation of cutaneous SCC by p53 tumor suppressor function was based on the observation that reductions in p53 typically do not increase the frequency or incidence of tumor formation but rather increase the frequency of malignant conversion to SCC (9). The current study confirms this traditional role for loss-of-function p53 mutations 